\section{Bestätigt: Viele sind vermeidbar}

\author{
Hypoglykämien sind vor allem bei älteren Typ-2-Diabetikern häufig, was \\ noch immer unterschätzt wird. Viele davon sind bei Entscheidung für \\ einen DPP-4-Hemmer statt eines Sulfonylharnstoffs vermeidbar. Das \\ hat eine aktuelle posthoc-Analyse bei älteren Patienten erneut belegt.
}

„Wie stark vor allem ältere Typ-2-Diabetiker zu Hypoglykämien neigen, wird allgemein unterschätzt“, sagte Prof. Anthony Barnett vom Diabetes Centre Heartlands Hospital in Birmingham/UK. Einer Studie aus dem Jahr 2008 zufolge treten bei bis zu 38\% der Patienten Hypoglykämie-Symptome auf [1], und dies betreffe natürlich auch Patienten mit oraler Medikation, so Barnett. Die Assoziation von Hypoglykämien mit verringerter Lebensqualität, Therapiezufriedenheit und Adherenz sowie mit erhöhter Morbidität und Mortalität machen sie zum ernsthaften Problem in der Diabetestherapie.

\section{Notrufe meist wegen Hypoglykämie} Um zu verdeutlichen, wie häufig Hypoglykämien im Alltag sind und wie uninformiert viele Patienten, stellte Barnett eine 2011 publizierte Untersuchung vor, für die Notrufdaten des East Anglia Ambulance Trust ausgewertet wurden. Etwa 2800 Notrufe innerhalb eines Jahres stan- den im Zusammenhang mit Diabetes mellitus. 85\% wurden aufgrund von $\mathrm{Hy}$ poglykämien getätigt, ,die meisten Betroffenen waren Typ-2-Diabetiker“, sagte Barnett. 44\% der Patienten gaben an, keine Warnsymptome einer Hypoglykämie gehabt zu haben, 28\% waren sich nicht darüber im Klaren, woher die Symptome kamen. 80\% der Notfallpatienten hatten seit mehr als 10 Jahren Diabetes, seien also bereits älter gewesen. 55\% hatten im vergangenen Jahr keine Schulung zu Hypoglykämien erhalten. „Diese Fakten sind beängstigend“, so Barnett. Dabei mache es auch keinen Unterschied, ob die Patienten Insulin oder Sulfonylharnstoffe (SH) erhalten, wie eine prospektive britische Studie ergeben habe [2]. Das Risiko für eine schwere Hypoglykämie bei SH-Therapie war mit 7\% ebenso groß wie bei Patienten, die weniger als 2 Jahre Insulin injizierten. Bei den milden symptomatischen Unterzuckerungen betrug das Risiko 39\% (SH) versus 51\% (Insulin).
Weniger Hypoglykämien mit Gliptin Welche Vorteile Sitagliptin (als Januvia, plus Metformin als Janumet ${ }^{\circledR}$ im Handel) im Vergleich zu den SH Glipizid und Glimepirid hat, verdeutlicht etwa eine posthoc-Analyse, die beim EASD in Berlin aktuell präsentiert worden ist [3]. Die gepoolten Daten entstammen drei doppelblinden klinischen Studien, in denen Typ-2-Diabetiker bis zu 104 Wochen lang das Gliptin $(100 \mathrm{mg} / \mathrm{d})$ oder einen der beiden SH jeweils in Monotherapie oder kombiniert zu Metformin erhalten hatten. Die Ergebnisse der 373 Patienten im Alter ab 65 Jahren nach etwa 30 Wochen Therapie: Bei der $\mathrm{HbA}_{1 \mathrm{c}}$-Reduktion (Basiswert 7,5\%) gab es mit-0,73\%- Punkten mit Sitagliptin $(\mathrm{n}=178)$ statistisch keinen signifikanten Unterschied zu den $\mathrm{SH}$ $\left(\mathrm{n}=195, \mathrm{HbA}_{1 \mathrm{c}}-078 \%\right.$-Punkte). Allerdings hatten $28 \%$ der SH-Patienten eine oder mehrere symptomatische Hypoglykämien, mit dem Gliptin waren es nur 6\%.

Sarah L. Pampel, München

\footnotetext{
1. Alvarez-Guisasola F et al. Diabetes Obes Metabol 2008 (10) Suppl. 1: 25-32.

2. UK Hypoglycaemia study group. Diabetologie 2007;50:1140-7.

3. Shankar R. et al. Diabetologie 2012;55 (Suppl.1) S.344, Abstract 834
}

Quelle: Symposium bei der EASD-Jahrestagung am 1.10.2012 in Berlin, Veranstalter MSD und MSD-Pressemeldung vom 2.10.2012

\title{
Rezepte von Ärzten für Hypertoniker
}

\section{Gesunde Ernährung hilft, bei Hypertonie den Blutdruck zu senken. Wie man gesund, lecker und einfach kocht, zeigt nun ein Kochbuch, das niedergelassene Kollegen für Hypertoniker verfasst haben.}

Die Allgemeinärztin Dr. Gerda Storm empfiehlt Hypertonikern Chicoree-Apfelsinen-Salat mit Bio-Joghurt. Die Internistin Dr. Alexandra Ramin setzt auf Winterkabeljau auf Süßkartoffelbett an Avocado-Tartar. Allgemeinmediziner Andreas Witzke gönnt seinen Patienten Rinderfilet auf Karotten-Vollkorn-Toast. Zusammen mit 50 anderen Ärzten sind sie Autoren eines von Novartis initiierten Kochbuches, mit dem Hypertonikern Appetit auf gesündere Ernährung ge- macht werden soll. Einfache, starke und individuelle Rezepte mit nur drei Zutaten lautete das Motto, in Anlehnung an die Attribute der Dreifachfixkombi Valsartan, Amlodipin und HCT (Exforge $\mathrm{HCT}^{\circledast}$ ), die fünf individuelle Dosierungen mit nur einer Tablette täglich ermöglicht. Die besten Rezepte ausgewählt hatte TVKoch Martin Baudrexel aus München. Gesunde Lebensführung mit ausgewogener Ernährung ist ein Grundpfeiler der Hypertonietherapie. Denn Übergewicht,

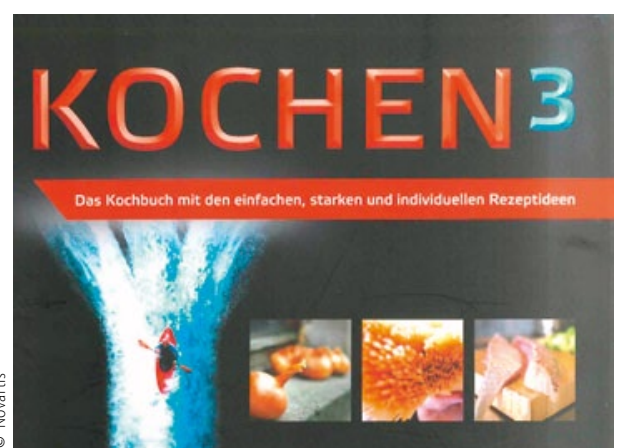

Das Kochbuch von Ärzten für Patienten mit Bluthochdruck: Rezepte mit 3 Hauptzutaten.

zu viel Kochsalz und Alkohol, zu wenig Kalium, falsche und zu viele Fette tragen zur Pathogenese bei, so Dr. Ursula Hildebrandt, Ernährungsmedizinerin aus Köln. Dr. med. Dirk Einecke, München

Quelle: Pressekonferenz beim ESC-Kongress in München, August 2012, Veranstalter: Novartis 